\title{
Hypoxia-inducible factor induces local thyroid hormone inactivation during hypoxic-ischemic disease in rats
}

\author{
Warner S. Simonides, ${ }^{1}$ Michelle A. Mulcahey, ${ }^{2}$ Everaldo M. Redout, ${ }^{1}$ Alice Muller, ${ }^{1}$ \\ Marian J. Zuidwijk, ${ }^{1}$ Theo J. Visser, ${ }^{3}$ Frank W.J.S. Wassen, ${ }^{3}$ Alessandra Crescenzi, ${ }^{2}$ \\ Wagner S. da-Silva, ${ }^{4}$ John Harney, ${ }^{4}$ Felix B. Engel, ${ }^{5}$ Maria-Jesús Obregon, ${ }^{6}$ \\ P. Reed Larsen, ${ }^{4}$ Antonio C. Bianco, ${ }^{4}$ and Stephen A. Huang ${ }^{2,4}$
}

\begin{abstract}
${ }^{1}$ Laboratory for Physiology, Institute for Cardiovascular Research, VU University Medical Center, Amsterdam, The Netherlands. ${ }^{2}$ Division of Endocrinology, Children's Hospital Boston, Boston, Massachusetts, USA. ${ }^{3}$ Department of Internal Medicine, Erasmus University Medical Center, Rotterdam, The Netherlands. 4Thyroid Section, Endocrinology, Diabetes, and Hypertension Division, Brigham and Women's Hospital, Boston, Massachusetts, USA.

${ }^{5}$ Department of Cardiology, Children's Hospital Boston, Boston, Massachusetts, USA. ${ }^{6}$ Unit of Molecular Endocrinology, Instituto de Investigaciones Biomédicas (CSIC-UAM) and CIBER Fisiopatologia de la Obesidad y Nutricion (CB06/03), Instituto de Salud Carlos III, Madrid, Spain.
\end{abstract}

\begin{abstract}
Thyroid hormone is a critical determinant of cellular metabolism and differentiation. Precise tissue-specific regulation of the active ligand 3,5,3'-triiodothyronine (T3) is achieved by the sequential removal of iodine groups from the thyroid hormone molecule, with type 3 deiodinase (D3) comprising the major inactivating pathway that terminates the action of $\mathrm{T} 3$ and prevents activation of the prohormone thyroxine. Using cells endogenously expressing D3, we found that hypoxia induced expression of the D3 gene DIO3 by a hypoxiainducible factor-dependent (HIF-dependent) pathway. D3 activity and mRNA were increased both by hypoxia and by hypoxia mimetics that increase HIF-1. Using ChIP, we found that HIF-1 $\alpha$ interacted specifically with the $D I O 3$ promoter, indicating that $D I O 3$ may be a direct transcriptional target of HIF-1. Endogenous D3 activity decreased T3-dependent oxygen consumption in both neuronal and hepatocyte cell lines, suggesting that hypoxia-induced D3 may reduce metabolic rate in hypoxic tissues. Using a rat model of cardiac failure due to RV hypertrophy, we found that HIF-1 $\alpha$ and D3 proteins were induced specifically in the hypertrophic myocardium of the RV, creating an anatomically specific reduction in local T3 content and action. These results suggest a mechanism of metabolic regulation during hypoxic-ischemic injury in which HIF-1 reduces local thyroid hormone signaling through induction of D3.
\end{abstract}

\section{Introduction}

During illness, humans experience a fall in serum thyroid hormones termed the nontbyroidal illness syndrome (1). An inverse relationship between serum thyroxine (T4) and mortality in critically ill patients has previously been observed (2), but several controlled trials have reported no benefit (3) or even worsened outcome (4) with thyroid hormone supplementation. This has led to the hypothesis that the nonthyroidal illness syndrome is an adaptive process that promotes survival during life-threatening illness by reducing metabolic rate and energy cost. Because illness is heterogenous, others postulate that this potential benefit is outweighed in certain patients by the impairment of 3,5,3'-triiodothyroninedependent (T3-dependent) cardiac output, respiratory drive, and renal function, and experts continue to debate the appropriateness of thyroid hormone treatment (5).

Most research of the nonthyroidal illness syndrome has focused on decreased T3 production as the cause of low serum T3 (6). Another potentially important regulatory mechanism is the peripheral inactivation of iodothyronines by deiodination. Type 1 deiodinase (D1) and D2 activate the prohormone T4 into

Nonstandard abbreviations used: CHF, congestive heart failure; D1, type 1 deiodinase; DFO, desferrioxamine; $5^{\prime} \mathrm{FR}, 5^{\prime}$ flanking region; HRE, hypoxia response element; rT3, reverse T3; T2, 3,3'-diiodothyronine; T3, 3,5,3'-triiodothyronine; T4, thyroxine. Conflict of interest: The authors have declared that no conflict of interest exists. Citation for this article: J. Clin. Invest. 118:975-983 (2008). doi:10.1172/JCI32824. the more biologically active T3 via outer-ring deiodination (7). In contrast, D3 is the physiologic inactivator of thyroid hormones, catalyzing the conversion of $\mathrm{T} 4$ and $\mathrm{T} 3$ to the inactive metabolites reverse T3 (rT3) and 3,3'-diiodothyronine (T2) via inner-ring deiodination. Although D3 is normally expressed in only a few postnatal tissues (8-10), its reactivation in the liver and skeletal muscle of critically ill patients has recently been reported and associated with decreases in serum T3 (11). Unlike other mechanisms, which lower T3 systemically by reducing its production, D3 has the unique potential to induce local hypothyroidism. This ability to induce anatomically and temporally precise hypothyroidism has been established in Xenopus laevis metamorphosis (12), but to our knowledge, a similar effect in postnatal D3-expressing mammalian tissues has not been previously shown. In addition, the molecular mechanisms responsible for D3's reactivation during illness are not known.

Because we recently discovered that the hypoxia target gene TGF- $\beta 3$ stimulates DIO3 transcription (13), we investigated hypoxia as a regulator of D3 expression. Several tissues that express D3 are known to be hypoxic, including the normal tissues of the human fetus (14) and the ischemic tissues of critically ill patients (11), but a molecular mechanism for this has not been established. Our present study shows that hypoxia increased D3 mRNA and activity in diverse cell types. Hypoxia-inducible factor (HIF) is the central transcriptional mediator of the cellular response to low oxygen, and hypoxia mimetics such as desferrioxamine (DFO) and $\mathrm{CoCl}_{2}$ were also sufficient to 


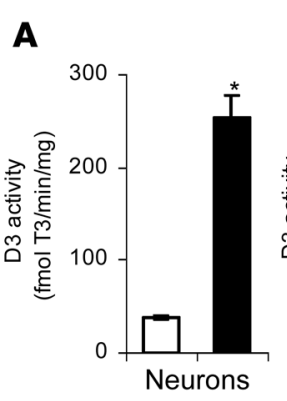

B

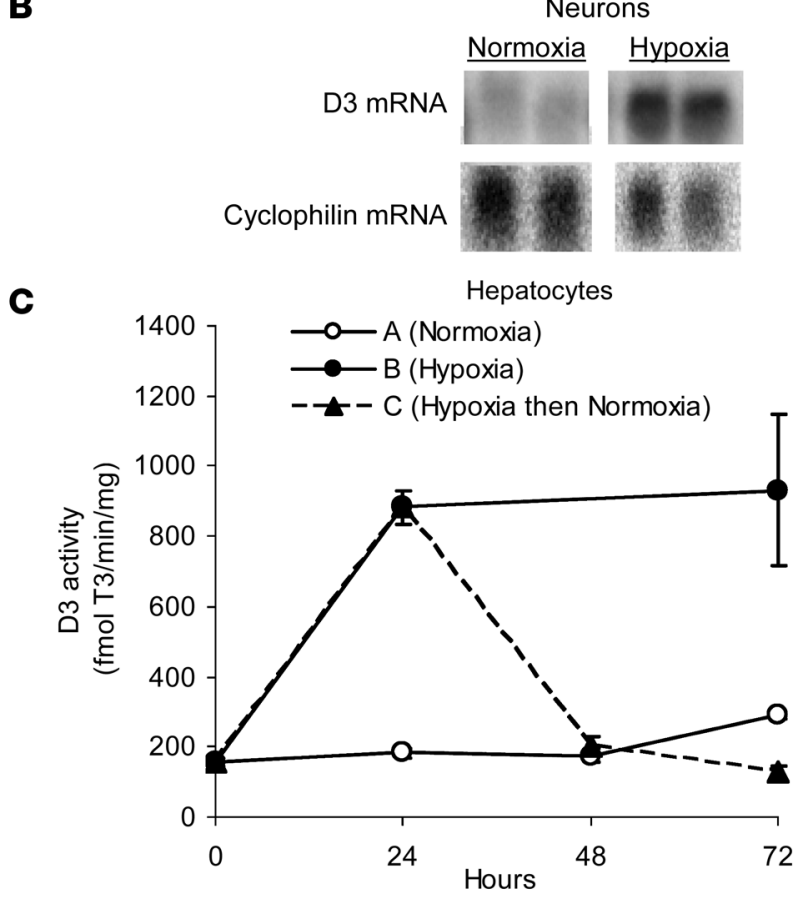

Hypoxia
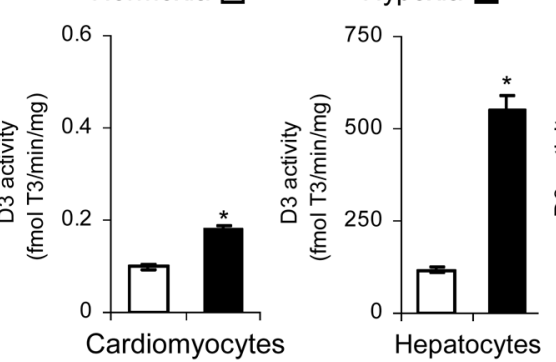

| 0 JEG-3 cells
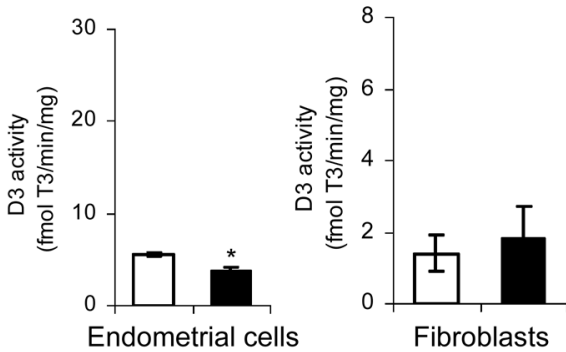

Hepatocytes

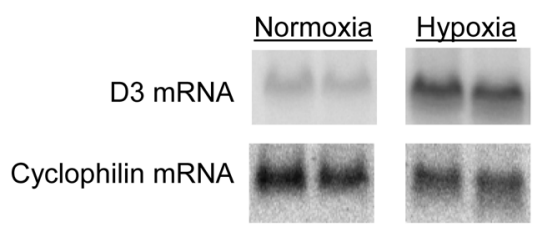

Hepatocytes Condition A (O) Condition C $(\boldsymbol{\Delta})$ $\underline{24 h} \underline{48 h} \quad 72 h \quad 24 h \quad 48 h \quad 72 h$

D3 mRNA

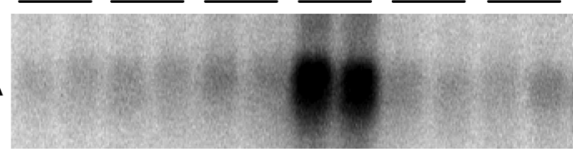

Cyclophilin mRNA

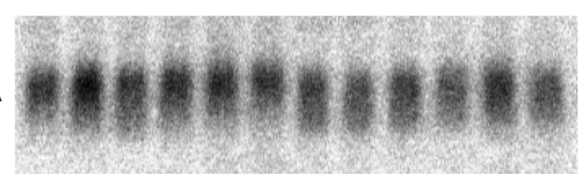

Figure 1

Hypoxia induces D3 activity and mRNA. (A) Endogenous D3 activity in SK-N-AS neurons, rat neonatal cardiomyocytes, NCLP6E hepatocytes, choriocarcinoma cells (JEG-3 cells), endometrial cells (ECC-1 cells), and AG04526 fibroblasts exposed to normoxia $\left(21 \% \mathrm{O}_{2}\right)$ versus hypoxia $\left(1 \% \mathrm{O}_{2}\right)$ for $24 \mathrm{~h}$. Values are mean \pm SEM of 2 or 3 cell plates; mean of 3 experiments is shown for each cell type. ${ }^{*} P<0.005$. (B) Northern blot analysis of total RNA obtained from SK-N-AS or NCLP6E cells exposed to hypoxia versus normoxia for $24 \mathrm{~h}$. Lanes were run on the same gel but were noncontiguous. (C) D3 activity and Northern blotting in NCLP6E cells exposed to continuous normoxia (condition A), continuous hypoxia (condition B), or transient hypoxia for $24 \mathrm{~h}$ followed by normoxia (condition $\mathrm{C}$ ). Representative experiment with mean \pm SEM of 2 cell plates is shown; this experiment was reproduced.

induce D3, indicating a HIF-dependent mechanism. This D3 activity was sufficient to inhibit T3-stimulated metabolic rate in isolated cells and to induce anatomically specific reductions in tissue $\mathrm{T} 3$ content and action in an in vivo model of RV hypertrophy. These data reveal a role of D3 in the regulation of local as well as systemic thyroid status during hypoxic-ischemic illness.

\section{Results}

Hypoxia induces sustained D3 expression. Multiple cell types were exposed to normobaric hypoxia ( $1 \%$ oxygen, $5 \% \mathrm{CO}_{2}, 94 \%$ nitrogen) versus normoxia ( $21 \%$ oxygen, $5 \% \mathrm{CO}_{2}, 74 \%$ nitrogen) for $24 \mathrm{~h}$, and endogenous D3 activity was measured using previously described HPLC methods (13). Hypoxia increased endogenous D3 activity in multiple cell types (Figure 1A), including neurons (human SK-N-AS cells; 5.6-fold), cardiomyocytes (isolated rat neonatal cardiomyocytes; 1.7-fold), hepatocytes (rhesus monkey NCLP6E cells; 4.8-fold), and choriocarcinoma cells (human JEG-3 cells; 18.7-fold). In contrast, hypoxia produced no significant increase in endogenous D3 activity in endometrial cells (human ECC-1 cells) or fibroblasts (human AG04526 cells), illustrating the cell type specificity of this phenomenon. To determine whether this D3 induction occurs at the level of RNA, SK-N-AS and NCLP6E cells were exposed to hypoxia, and after $24 \mathrm{~h}$ the D3/cyclophilin ratio in hypoxic cells was 5.2- and 7.8-fold higher, respectively, than that in normoxic controls (Figure 1B). This experiment was repeated with cells endogenously expressing D1 (GC-1 cells) or D2 (MSTO), and no change in D1 or D2 enzyme activity was seen (data not shown). This concept that the response of specific deiodinases to hypoxia is variable between cell types is further supported by the recent report of increased D2, but not D3, activity in hypoxic rat astrocytes (15).

To determine whether this high D3 expression could persist with chronic hypoxia, we next exposed NCLP6E hepatocytes to 
A
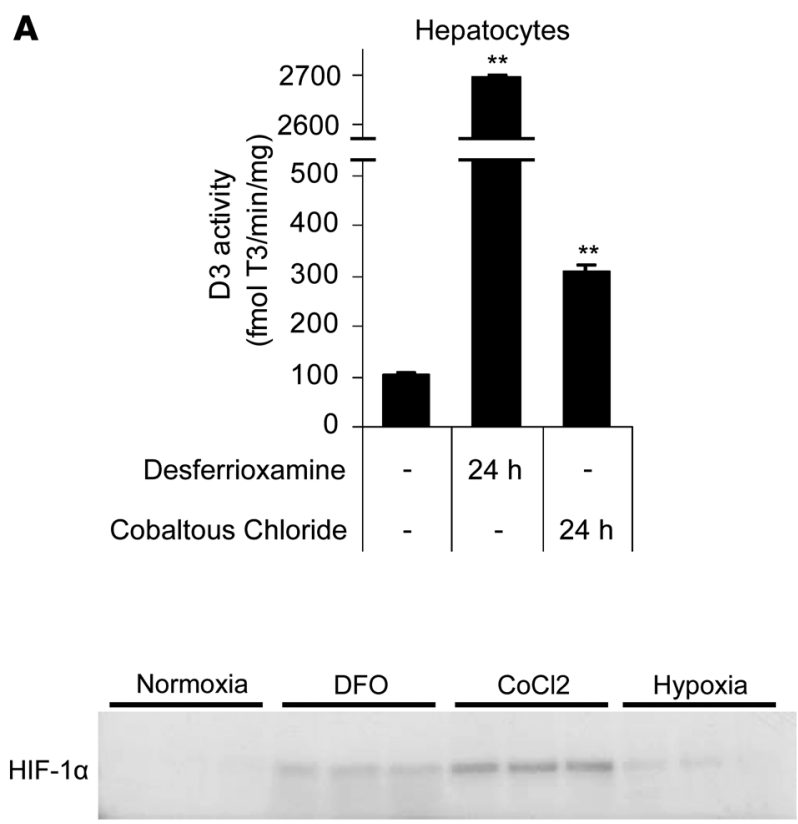

C
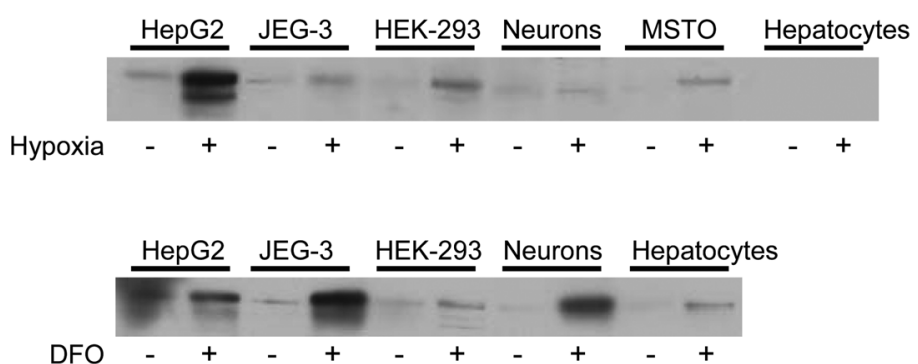

B

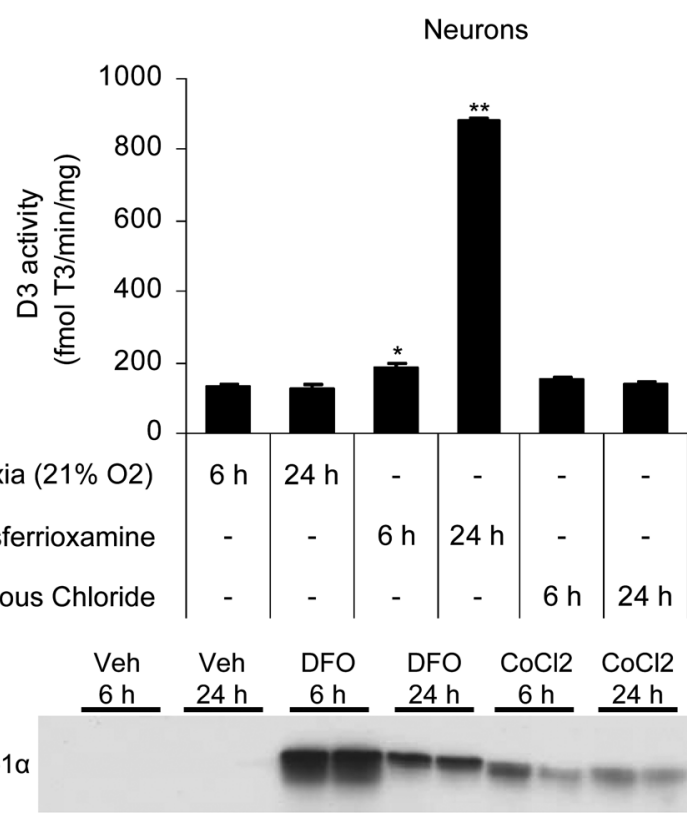

D

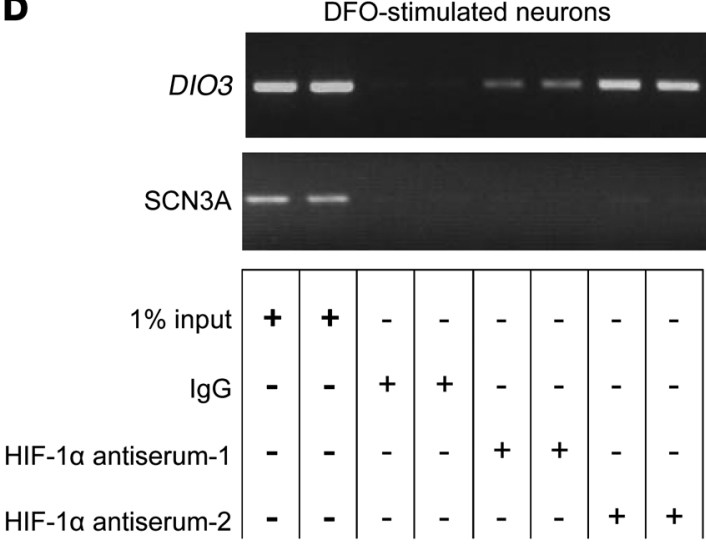

\section{Figure 2}

The hypoxia mimetics DFO and $\mathrm{CoCl}_{2}$ increase endogenous D3 in NCLP6E hepatocytes and SK-N-AS neurons. (A) D3 activity and Western blot analysis of HIF-1 $\alpha$ protein in NCLP6E hepatocytes $24 \mathrm{~h}$ after exposure to the indicated hypoxia mimetics. Hepatocytes that were exposed to hypoxia (without hypoxia mimetics) are included in the HIF-1 $\alpha$ Western blot as a positive control. (B) D3 activity and Western blot analysis of HIF-1 $\alpha$ protein in SK-N-AS neurons 6 or $24 \mathrm{~h}$ after exposure to DFO or $\mathrm{CoCl}_{2}$. Values are mean \pm SEM of 3 cell plates. ${ }^{*} P<0.05 ;{ }^{* *} P<0.005$. (C) Western blot analysis of HIF-1 $\alpha$ protein in cells that increase endogenous D3 expression in response to hypoxia (JEG-3, SK-N-AS neurons, and NCLP6E hepatocytes) and in cells with undetectable endogenous D3 activity (HepG2, HEK-293 cells, and MSTO cells). (D) ChIP analysis of the DIO3 or the SCN3A promoter in DFO-stimulated SK-N-AS cells using antibodies directed against endogenous HIF-1 $\alpha$. Representative experiments are shown and were reproduced.

continuous normoxia for $72 \mathrm{~h}$, continuous hypoxia for $72 \mathrm{~h}$, or transient hypoxia for $24 \mathrm{~h}$ followed by normoxia. By $24 \mathrm{~h}$, D3 activity in hypoxic NCLP6E cells was 4.8-fold higher than in normoxic controls; with continuous hypoxia, D3 activity remained elevated to the last time point tested (Figure 1C). This was in contrast to the temporary peak of D3 activity previously reported with other stimulators such as phorbol ester and the fibroblast growth factors $(13,16)$. In cells transiently exposed to hypoxia, the cessation of hypoxia was followed by the rapid return of stimulated D3 activity and message to normoxic levels (Figure 1C), indicating that the modulation of D3 by oxygen tension is dynamic rather than an irreversible stimulation initiated by hypoxic stress.
Hypoxia mimetics induce D3. Hypoxia is the physiologic trigger that activates HIF, and HIF is also upregulated in vitro by hypoxia mimetics such as $\mathrm{CoCl}_{2}$ and DFO, which inhibit HIF ubiquitination and lead to its accumulation under even normoxic conditions (17). To assess HIF dependence, we exposed normoxic NCLP6E hepatocytes to $\mathrm{CoCl}_{2}$ and DFO, which increased endogenous D3 activity 3.0- and 26.3-fold, respectively (Figure 2A). HIF- $1 \alpha$ accumulation was confirmed by Western blot. In SK-N-AS neurons, HIF- $1 \alpha$ protein was detectable by Western blot as early as $6 \mathrm{~h}$ after DFO administration, and D3 activity was 7.0-fold higher by $24 \mathrm{~h}$ (Figure 2B). These data indicate that HIF accumulation (even without hypoxia) is sufficient to stimulate D3 expression and is strong evidence that hypoxia induces D3 via the HIF pathway. At 
A
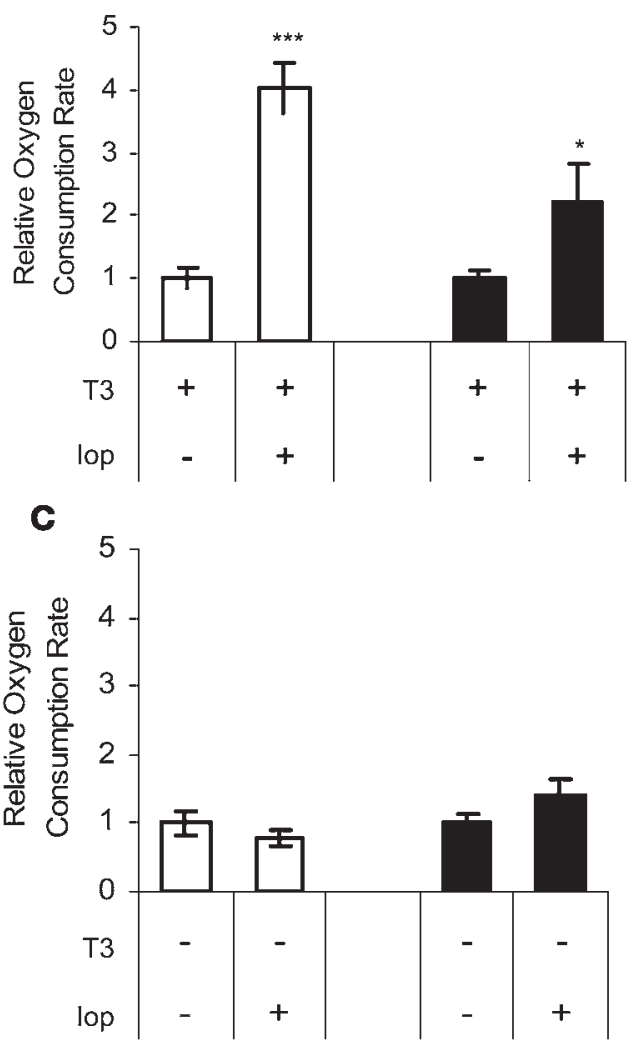
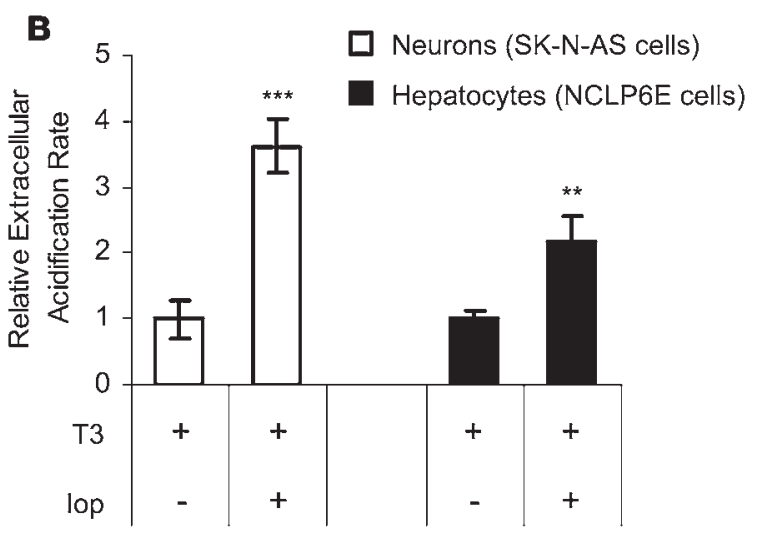

D

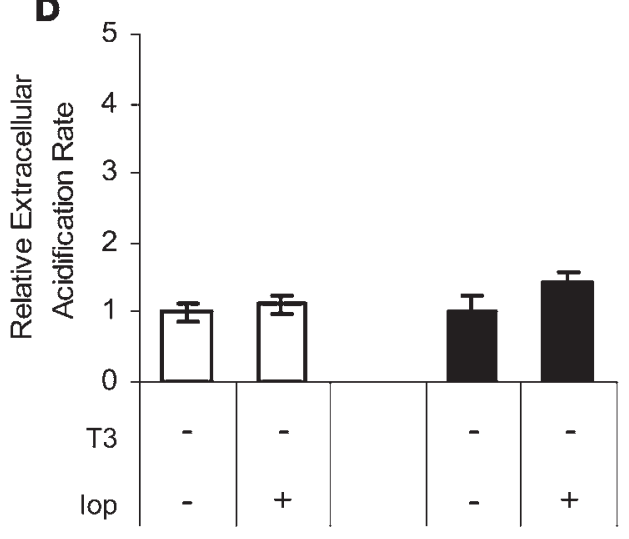

Figure 3

Inhibition of endogenous D3 activity with iopanoic acid for $6 \mathrm{~h}$ increases metabolic rate in isolated cells. Oxygen consumption (A and C) and extracellular acidification (B and D) in SK-N-AS neurons and NCLP6E hepatocytes that were propagated in media with (A and B) or without (C and D) a euthyroid concentration of T3 and exposed to iopanoic acid (lop) versus vehicle control. Oxygen consumption rate (pmol/min/mg protein) and extracellular acidification rate $(\mathrm{mpH} / \mathrm{min} / \mathrm{mg}$ protein) are expressed as relative fold change from vehicle control. Values are mean \pm SEM of 10 cell plates. ${ }^{*} P=0.07 ;{ }^{* \star} P<0.05 ;{ }^{* \star} P<0.005$.

the limited time points tested, D3 activity was not consistently proportional to mimetic-induced HIF-1 accumulation, reflecting differences in the half-lives of HIF-1 versus D3 protein and perhaps variable affinity of the antiserum for monkey (NCLP6E hepatocytes) versus human (SK-N-AS neurons) HIF-1 $\alpha$ protein. D3 induction in response to either hypoxia- or DFO-induced HIF-1 was variable across different cell types, with no measurable D3 activity in stimulated HepG2, HEK-293, or MSTO (18) cells despite strong HIF-1 induction (Figure 2C). This indicates that HIF accumulation is necessary but not sufficient to induce D3 during hypoxia and that other cell type-specific factors are required for high D3 expression.

HIF transactivates target genes by binding 1 or more hypoxia response elements (HREs). To assess the possibility of HREdependent Dio3 stimulation, we used ChIP analysis to examine the association of HIF- $1 \alpha$ with a conserved HRE in the 5 ' flanking region $\left(5^{\prime} \mathrm{FR}\right)$ of the $\mathrm{DIO} 3$ gene $(-832$ bp relative to the DIO3 transcription start site). Human SK-N-AS neurons were exposed to DFO under conditions that induce both HIF- $1 \alpha$ and endogenous D3 (Figure 2B). Chromatin was immunoprecipitated with 2 different HIF- $1 \alpha$ antibodies, using preimmune IgG as a negative control. Notably, an appropriately sized amplicon was amplified only from chromatin immunoprecipitated with either HIF- $1 \alpha$ antiserum (Figure 2D), indicating that HIF- $1 \alpha$ interacts specifically with these $\mathrm{DIO} 3$ sequences. As an additional negative control, PCR was repeated on these same specimens using primers directed to the promoter of an unrelated gene, the human SCN3A sodium channel (19). As expected, no SCN3A amplicon was observed in samples immunoprecipitated with either HIF-1 $\alpha$ antisera or preimmune IgG.

Inbibition of D 3 catalytic activity increases metabolic rate in isolated cells. We next sought to determine whether the endogenous D3 expression stimulated by hypoxia could lead to cellular hypothyroidism and alter a thyroid hormone-dependent phenotype. Because T3 is known to stimulate metabolic rate, we reasoned that D3 expression could decrease energy expenditure and, if so, that its induction during hypoxia could reduce oxygen consumption. To test this, we exposed SK-N-AS neurons and NCLP6E hepatocytes to iopanoic acid, a competitive inhibitor of iodothyronine deiodination, or vehicle control (DMSO). These cells were chosen because their basal D3 expression approximates the physiologic D3 activity of a normal human tissue, term placenta. Cells were grown in media containing charcoal-stripped FBS that was supplemented to a euthyroid concentration $(150 \mathrm{ng} / \mathrm{dl})$ of $\mathrm{T} 3$ or with vehicle without $\mathrm{T} 3$ prior to media preparation. The iopanoic acid concentration used $(20 \mu \mathrm{M})$ was optimized in prior experiments to inhibit cellular deiodination by whole-cell in situ deiodination assay (ref. 13 and data not shown). 
A

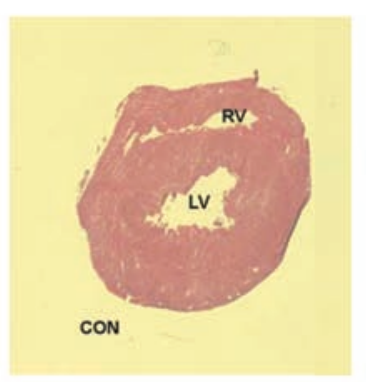

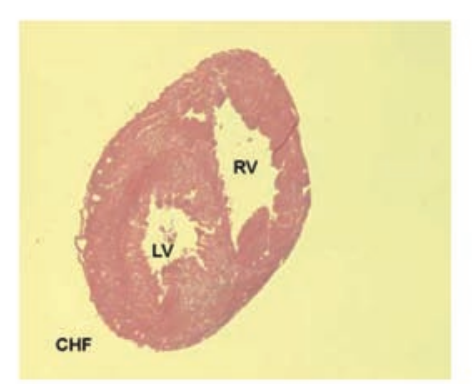
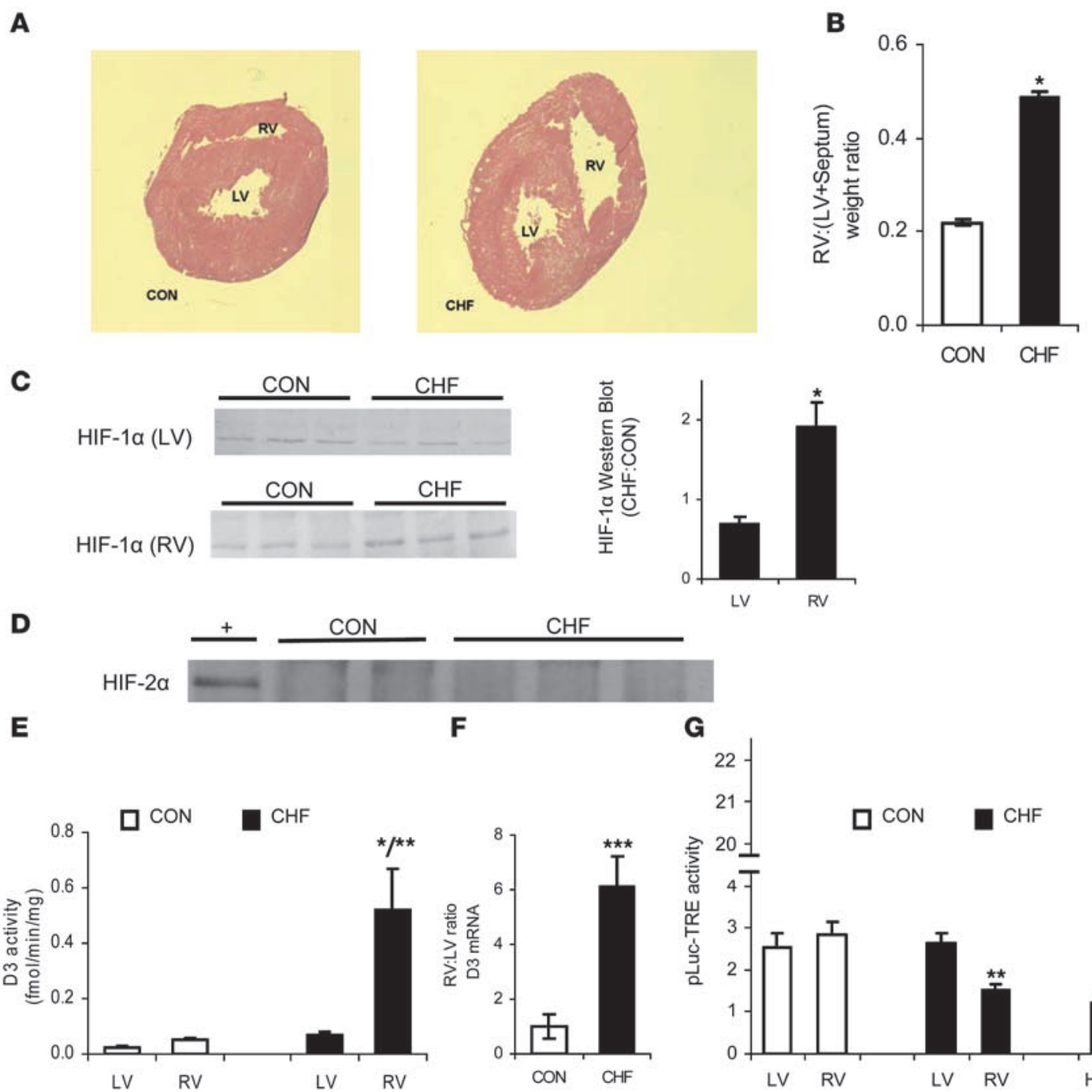

$\mathrm{CHF}$
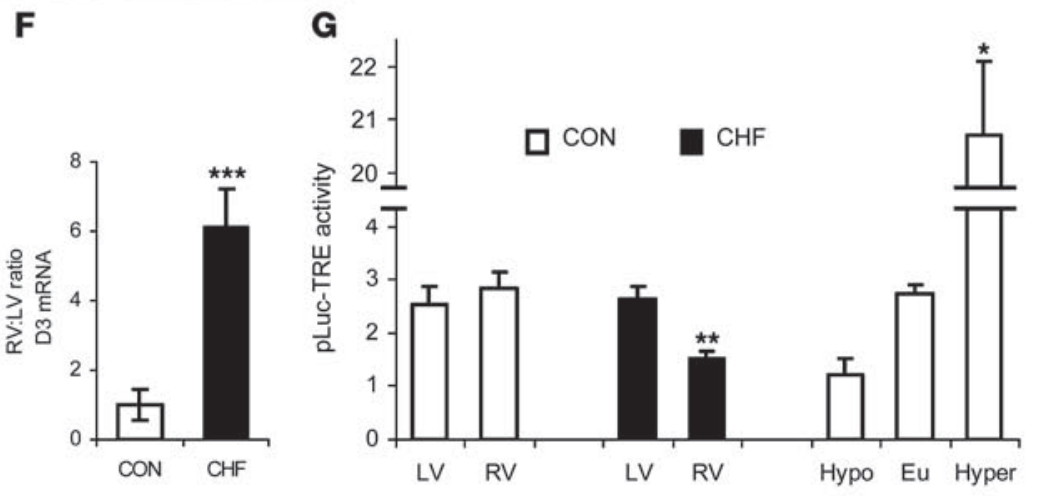

Figure 4

HIF-1 $\alpha$ and D3 are selectively induced in the RV in a rat model of monocrotaline-induced RV hypertrophy. Thickening of the RV wall (A) and increased RV weight (B) were observed as CHF developed after monocrotaline administration. Values are mean \pm SEM of 11-16 animals per group. (C-F) Western blot analysis of HIF-1 $\alpha(\mathbf{C})$ and HIF-2 $\alpha$ protein (D), D3 activity (E), and quantitative real-time PCR of D3 mRNA (F) in tissue prepared from the RV and LV of rats administered monocrotaline (CHF) versus saline control (CON). The HIF-2 $\alpha$ Western blot in D depicts RV samples from control and CHF animals, with extracts of primary microvascular endothelial cells obtained from human foreskin and cultured under hypoxic conditions $\left(1 \% \mathrm{O}_{2}\right)$ serving as a positive control $(+)$. Values are mean \pm SEM of 3-9 animals per group. (F) D3 mRNA is expressed as the RV/LV ratio and shown as the relative fold change from the saline control group. ${ }^{*} P<0.05$ versus control; ${ }^{* *} P<0.05$ versus $\mathrm{LV} ;{ }^{* \star *} P<0.005$ versus control. (G) Myocardial reporter activity after in vivo cardiomyocyte transfection of the pLuc-TRE T3-responsive Firefly luciferase reporter, normalized to the expression of the pRen-C transfection control. Values are mean \pm SEM of 5-29 animals per group. LV and RV reporter activity is shown in CHF versus control animals. In control animals with systemic hypothyroidism (Hypo), euthyroidism (Eu), or thyrotoxicosis from T3 treatment (Hyper), reporter levels from pooled LV/RV homogenates are shown. ${ }^{*} P<0.05$ versus euthyroidism or hypothyroidism, ANOVA; ${ }^{\star \star} P<0.05$ versus control and LV.

Cellular energy expenditure was analyzed with a recently developed noninvasive technology that uses a fluorophore-containing ruthenium complex to measure $\mathrm{O}_{2}$ consumption for approximately 20,000 intact cells during repeated 5-min intervals (20). The rate of extracellular $\mathrm{pH}$ change was measured simultaneously as an index of glycolytic flux. After only 6 h, D3 inhibition with iopanoic acid resulted in an increase in oxygen consumption and extracellular acidification. In the presence of T3, the oxygen consumption rate increased 4.0- and 2.2-fold and the extracellular acidification rate increased 3.6- and 2.2-fold in SK-N-AS and
NCLP6E cells, respectively (Figure 3, A and B). In the absence of T3 (media prepared with charcoal-stripped FBS without T3 supplementation), iopanoic acid produced no significant change in these same indices (Figure 3, C and D), confirming that these effects are thyroid hormone dependent.

$H I F-1 \alpha$ and D3 are induced in a rat model of RV bypertrophy and associated with an anatomically precise reduction in tissue T3 content and T3-dependent transcriptional activity. We have recently reported the induction of D3 in a rat model of monocrotaline-induced RV hypertrophy, but the mechanism of D3 induction in this system 
Table 1

Systemic and local thyroid status are partially discordant in a rat model of RV hypertrophy

\begin{tabular}{|c|c|c|c|c|}
\hline & \multicolumn{2}{|c|}{ Control } & \multicolumn{2}{|c|}{ CHF } \\
\hline Plasma T4 (nM) & \multicolumn{2}{|c|}{$55.0 \pm 7.0(9)$} & \multicolumn{2}{|c|}{$21.0 \pm 0.2(11)^{A}$} \\
\hline Plasma T3 (nM) & \multicolumn{2}{|c|}{$0.94 \pm 0.14(9)$} & \multicolumn{2}{|c|}{$0.36 \pm 0.05(11)^{\mathrm{A}}$} \\
\hline & LV & RV & LV & RV \\
\hline Tissue T4 (ng/g wet wt) & $6.39 \pm 0.52(5)$ & $6.34 \pm 0.35(5)$ & $2.44 \pm 0.17(6)$ & $2.86 \pm 0.10(6)$ \\
\hline Tissue T3 (ng/g wet wt) & $1.94 \pm 0.12(6)$ & $1.80 \pm 0.05(5)$ & $0.60 \pm 0.06(7)^{\mathrm{A}}$ & $0.39 \pm 0.04(7)^{A, B}$ \\
\hline Tissue T3/plasma T3 ratio & $2.04 \pm 0.21(6)$ & $1.90 \pm 0.12(5)$ & $1.52 \pm 0.27(7)^{\mathrm{A}}$ & $1.08 \pm 0.18(7)^{A, B}$ \\
\hline
\end{tabular}

Plasma and tissue thyroid hormone concentrations are mean $\pm \mathrm{SEM}$ of the number of experiments (shown in parentheses). ${ }^{\mathrm{A} P}<0.05$ vs. control. ${ }^{\mathrm{B} P}<0.05 \mathrm{vs}$. LV.

was unknown (21). Because myocyte hypoxia can occur in hypertrophic failure as myocyte diameter increases and oxygen diffusion decreases $(22,23)$, we investigated the possibility that HIF was involved. Rats were administered a single dose of monocrotaline to induce pulmonary hypertension and concentric RV hypertrophy, which then progresses to congestive heart failure (CHF). All animals in the monocrotaline group developed CHF, as evidenced by weight loss, pleural effusions, and ascites. RV hypertrophy was confirmed by thickening of the RV wall (Figure 4A) and by a 2.2-fold increase in the weight ratio of the RV to the LV and septum (Figure 4B). In the monocrotaline group, HIF-1 $\alpha$ protein increased 1.9-fold $(P<0.05)$ in the hypertrophic RV compared with the LV (Figure 4C). At the same time, no significant differences in HIF- $1 \alpha$ protein were seen between the RV and LV in the saline control group (Figure 4C), and HIF-2 $\alpha$ was undetectable in all samples (Figure 4D). D3 followed the same pattern of distribution as HIF-1 $\alpha$, with high D3 activity only in the hypertrophic RV of rats in the monocrotaline group (Figure 4E). As in isolated cells, the 9-fold increase in D3 activity was associated with high levels of D3 mRNA, with a 6.2-fold increase in the RV/LV ratio detected by real-time PCR in the monocrotaline group (Figure 4F). D2 activity was undetectable in all specimens (data not shown).

To analyze the impact of this myocardial D3 expression on local thyroid status, we measured tissue thyroid hormone concentrations (Table 1). Monocrotaline-induced CHF was associated with a $35 \%$ drop $(P<0.05)$ in T3 concentration in the hypertrophic RV relative to the LV of the same hearts, corresponding to D3's anatomic distribution, whereas the T4 content was the same in both ventricles. In contrast, no difference in tissue T3 or $\mathrm{T} 4$ content was observed between the RV and LV of rats in the control group. While the decrease in plasma T3 and T4 noted in the monocrotaline group contributes to overall lower tissue hormone levels, it cannot explain the discordance between LV and RV T3 concentrations in this group. This was illustrated by a specific decrease in the tissue $\mathrm{T} 3$ to plasma $\mathrm{T} 3$ ratio in the $\mathrm{D} 3$ expressing hypertrophic RV (Table 1 ).

We next tested the physiologic relevance of this apparent local hypothyroidism using what we believe to be a novel method to measure T3-dependent transcriptional activity in vivo. A T3-responsive Firefly luciferase vector (pLuc-TRE) and a normalization Renilla luciferase vector ( $\mathrm{pRen}-\mathrm{C}$ ) were constructed and coinjected into the myocardium via thoracotomy to selectively transfect cardiomyocytes $(24,25)$. Reporter activity was assayed in ventricular homogenates 5 days later. The T3 responsiveness of this system was validated by comparing myocardial pLuc-TRE activity in rats that were euthyroid, systemically hypothyroid $\left(\mathrm{KClO}_{4}\right.$ administration), or thyrotoxic (T3 administration) (Figure 4G). In monocro- taline-treated animals, T3-dependent transcriptional activity was reduced by $45 \%(P<0.05)$ only in the D3-expressing RV, to levels that approximate those observed in the myocardium of systemically hypothyroid animals despite euthyroid reporter levels in the LVs of the same hearts (Figure 4G). Taken together, these data indicate that both HIF- $1 \alpha$ and D3 are selectively induced in the $\mathrm{RV}$ in this model of hypertrophic CHF and that this D3 activity is associated with a local anatomically precise reduction of T3 as well as T3-dependent transcriptional activity.

\section{Discussion}

D3-mediated inner ring deiodination was originally characterized in the placenta $(26,27)$ and in embryonic tissues $(28)$, where D3's highly coordinated expression permits the spatially and temporally precise inactivation of thyroid hormone during morphogenesis (12). Although D3 is not detectable in most normal postnatal tissues, recent studies have documented D3 reactivation in various disease states and indicated that its degradation of circulating thyroid hormone can lower serum T3 (11) or even cause severe hypothyroidism (29). These phenomena illustrate D3's clinical relevance as a regulator of systemic thyroid status, but the molecular mechanisms responsible for its reactivation and the functional consequences to the tissue microenvironment are largely unknown. Here we show that hypoxia induced D3 (Figure 1) via a HIF-dependent pathway (Figure 2) and demonstrate that D3 activity was sufficient to reduce T3-stimulated energy expenditure (Figure 3 ) and to induce anatomically specific local hypothyroidism in hypoxic tissue (Figure 4 and Table 1).

In cells endogenously expressing D3, both hypoxia (Figure 1) and hypoxia mimetics (Figure 2) were sufficient to induce high D3 activity, indicating that D3's induction is HIF dependent. This D3 induction was regulated at the level of RNA (Figure 1B), and ChIP analysis of stimulated human neurons indicated that HIF-1 $\alpha$ interacted specifically with sequences in the DIO3 5'FR (Figure 2D), thus providing strong evidence that D3 is a direct HIF-1 target gene. HIF-1 $\alpha$, but not HIF-2 $\alpha$, was induced in the D3-expressing RV of monocrotaline-treated rats, indicating that HIF- $1 \alpha$ is the stimulating HIF isoform in this model of CHF (Figure 4, $\mathrm{C}$ and D). Whether or not HIF-2 $\alpha$, a closely related isoform of HIF- $1 \alpha$ that has recently been shown to play nonredundant cell type-specific roles in hypoxic responses such as erythropoietin induction $(30,31)$, can also mediate the hypoxic induction of D3 in certain other tissues or developmental states remains to be determined. Adding further complexity, because hypoxia has also been shown previously to induce factors such as TGF- $\beta 3$ and basic fibroblast growth factor (32) that are also capable of stimulating D3 (13), indirect D3 stimulation via these intermediates in certain cell 
types is also possible. Further characterization of these potential signaling interactions may explain the variation in D3 response we observed among cell types (Figure 1A), but the present study clearly demonstrated that hypoxia stimulated D3 in a temporally dynamic (Figure 1C) and anatomically specific manner (Figure 4 and Table 1). The ability of D3 to decrease metabolic rate and oxygen consumption (Figure 3 ) is consistent with the function of many other HIF target genes (17), and this supports the general concept that $\mathrm{T} 3$ reduction during nonthyroidal illness benefits energy balance. D3 can induce spatially precise local hypothyroidism (Table 1) because it is an integral plasma membrane protein, thus decreasing the access of $\mathrm{T} 3$ to the cell nucleus and consequent binding to $\mathrm{T} 3$ receptors (33). As illustrated in the rat CHF model in the present study, D3 maintained local T3 gradients across subpopulations of cells even within the same tissue or organ (Table 1) and induced anatomically precise reductions in thyroid hormone-dependent transcription (Figure 4G). The role of D3 is the opposite of that played by D2, the key thyroid hormone-activating enzyme present in the endoplasmic reticulum compartment (34) that can increase T3 concentration within the nucleus and thus saturation of $\mathrm{T} 3$ receptors (35).

The induction of D3 by hypoxia can explain several clinical observations. D3 is highly expressed throughout the human fetus but falls to undetectable levels in all but a few tissues within a few days of delivery $(8,9)$. This coincides with the transition from fetal to neonatal circulation and the rapid rise in the partial pressure of arterial oxygen from $25 \mathrm{mmHg}$ in the fetus (36) to $74 \mathrm{mmHg}$ in the newborn infant (37) as the ductus arteriosus and the foramen ovale close. Children who are chronically hypoxic from pathologic conditions such as congenital heart disease also have a pattern of serum iodothyronines that is consistent with the accelerated innerring deiodination of T4 (high TSH, low T4, high rT3; ref. 38). Even in patients who die in the intensive care unit, D3-specific activity in the liver and skeletal muscle correlates positively with inotrope requirements, suggesting that tissue ischemia stimulates higher D3 expression and a greater fall in serum T3 (11). D3 stimulation by HIF can explain the modulation of systemic thyroid status in all of these hypoxic-ischemic conditions. In addition, the current study also provides insight into the impact of postnatal D3 expression on the tissue microenvironment. Although the massive D3 reactivation in conditions such as consumptive hypothyroidism is clearly maladaptive, the observation that D3 reduces T3-dependent oxygen consumption suggests that D3 reactivation in certain other disease states may benefit the patient. Using the example of hypertrophic cardiac failure, local D3 expression could promote viability in the most hypoxic myocytes by reducing oxygen consumption without a global decrease in T3-dependent contractility in the healthy myocardium. Further studies are needed to determine the role of D3 reactivation in clinical disease, but because SK-N-AS neurons, cardiomyocytes, and NCLP6E hepatocytes all increased D3 in responses to hypoxia (Figure 1A), we speculate that local thyroid hormone inactivation by $\mathrm{D} 3$ may be an important component of the tissue response to diverse hypoxic-ischemic injuries such as stroke, myocardial infarction, and liver transplantation.

From a broad perspective, our findings are reminiscent of the cell-specific modulation of thyroid hormone signaling by D2. It has long been established that the expression of D2 in tissues such as the pituitary (39), brain (40), and brown fat (41) can amplify local thyroid hormone signaling through the intracellular activation of T4 into T3. Here we show an opposite but comple- mentary physiologic role of D3 to inactivate thyroid hormones and mediate cell-specific hypothyroidism. It is accepted that D3 serves a homeostatic role in tissues such as the brain to counteract changes in thyroid hormone supply and maintain tissue euthyroidism during thyroid dysfunction (42). In addition, D3 inactivation disrupts thyroid hormone homeostasis and leads to central hypothyroidism (43). Studies have shown that primary changes in D2 can change thyroid status, and we show here that D3 induction by the hypoxia-HIF signaling pathway decreased local T3 concentrations (Figure 4 and Table 1) and cell-specific thyroid hormone-dependent metabolic effects (Figure 3), thus adding to the growing body of work that indicates a fascinating role of deiodination in postnatal disease as well as in embryonic development and thyroid hormone homeostasis.

\section{Methods}

Materials. $\mathrm{CoCl}_{2}$, DFO, FBS, iopanoic acid, and sodium selenite were purchased from Sigma-Aldrich. SK-N-AS cells were provided by R. George (Dana Farber Cancer Institute, Boston, Massachusetts, USA). Neonatal rat cardiomyocytes were previously generated in our laboratory (44). Fibroblast cultures were purchased from the Coriel Cell Repository. Studies of human tissues were approved by the Investigative Review Boards of the Brigham and Women's Hospital and Children's Hospital Boston.

Tissue culture. SK-N-AS neurons were propagated in RPMI media with $10 \%$ FBS. Rat neonatal cardiomyocytes were grown in DMEM/F12 (Invitrogen) supplemented with $3 \mathrm{mM}$ sodium pyruvate, $0.2 \% \mathrm{BSA}, 0.1 \% \mathrm{mM}$ ascorbic acid, $0.5 \%$ insulin-transferrin-selenium (100x), penicillin, streptomycin, and L-glutamine (44). NCLP6E Macaca mulatta hepatocytes and AG04526 fibroblasts were propagated in MEM supplemented with $0.5 \%-10 \%$ FBS, glutamine, and gentamycin. Media for all cell types was supplemented with $10^{-7} \mathrm{M}$ sodium selenite. For experiments measuring metabolic rate, FBS was charcoal stripped prior to media preparation and cells were exposed to $20 \mu \mathrm{M}$ iopanoic acid versus DMSO vehicle. Cells were exposed to hypoxia versus normoxia in closed hypoxia chambers (Billups-Rothenberg Inc.).

Deiodination assays. Cellular sonicates were prepared for enzyme analysis using $0.1 \mathrm{M}$ phosphate and $1 \mathrm{mM}$ EDTA at $\mathrm{pH} 6.9$ with $10 \mathrm{mM}$ dithiothreitol and $0.25 \mathrm{M}$ sucrose. D1 and D2 assays were performed as previously described using 3,3', $\left.{ }^{125} \mathrm{I}\right] 5^{\prime}$-rT3 and 3,5,3', $\left[{ }^{125} \mathrm{I}\right] 5^{\prime}$-T4 (Perkin Elmer), respectively (45). D2 assays were performed in $100 \mathrm{nM} \mathrm{T} 3$ to saturate coexistent D3 activity. D3 activity was assayed by HPLC as previously described using 1-150 $\mu$ g cellular protein, 200,000 cpm 3,5,[125 I] 3'-T3 (Perkin Elmer), $1 \mathrm{mM} 6 \mathrm{~N}$-propylthiouracil, $10 \mathrm{mM}$ DTT, and $0-10 \mathrm{nM}$ unlabeled T3 in each reaction. Reactions were stopped by the addition of methanol, and the products of deiodination were quantified by HPLC as described by Richard et al. (9). D3 velocities are expressed as fmol of T3 inner-ring deiodinated per $\mathrm{mg}$ of sonicate protein per minute ( $\mathrm{fmol} / \mathrm{mg} / \mathrm{min}$ ).

Northern blotting. Total RNA was isolated with TRIzol reagent (Invitrogen). Northern blots were prepared according to standard methods and probed with a 1.9-kb fragment representing the full length of the DIO3 cDNA (27). D3 Northern blotting was performed using 15-30 $\mu \mathrm{g}$ total RNA exposed for 3 days on a phosphoimager. Blots then were stripped and reprobed with a rat cyclophilin cDNA probe to adjust for differences in sample loading and transfer. Consistent with the high degree of homology between monkey and human D3 genes predicted from the rhesus Macaque Genome project, D3 Northern blotting of NCLP6E cells confirmed the presence of a single D3 mRNA band that comigrated with transcript from human term placenta.

ChIP assay. ChIP assays were performed according to the Upstate EZ ChIP kit protocol (Millipore). SK-N-AS cells were exposed to DFO for $24 \mathrm{~h}$ and then exposed to $1 \%$ formaldehyde to crosslink the chromatin prior 
to shearing. Sheared chromatin was immunoprecipitated with rabbit polyclonal antibodies against HIF-1 $\alpha$ from either Santa Cruz Biotechnology Inc. or Abcam Inc., using preimmune rabbit IgG (Santa Cruz Biotechnology Inc.) as a negative control. The primer sequences used to amplify an HRE-containing region of the DIO3 5'FR were as follows: forward, 5'-TCCGGCGTTCAGGCAACAGCTA-3'; reverse, 5'-CCGTCCCATCTCGTTCCA-3'. Primers used to amplify the SCN3A sodium channel promoter were previous described (19).

Cellular metabolic rate. An XF24 Extracellular Flux Analyzer (Seahorse Bioscience) was used to measure oxygen consumption rates in real time. Cells were trypsinized, counted, and then seeded in customized 24-well plates, each with $28 \mathrm{~mm}^{2}$ surface and $350 \mu \mathrm{l}$ media. SF 24 optical sensors coupled to fiber optic wave guides were used to deliver light at various excitations wavelengths and transmit a fluorescent signal to photo detectors. Fibers were coupled through a set of narrow band pass optical filters to separate the wavelengths specific to each probe's excitation and emission characteristics, and oxygen concentration was measured as the quenching of fluorescence from a porphyrin-based fluorophore specific to oxygen embedded in the polymer of a disposable cartridge. Analyzer probes were contained in a vertically mobile probe head that was lowered onto the wells during each measurement cycle. Lowering the probe head reduced the volume of analysis to approximately $7 \mu \mathrm{l}$ of media. Oxygen consumption rate was measured during $5 \mathrm{~min}$, after which the probe head was lifted and the cells exposed to fresh media. This cycle was repeated 4 times at 5 -min intervals. Simultaneous measurements of $\mathrm{pH}$ were obtained in parallel.

Animals. Animal care and experiments were approved by the IACUC of the VU University Medical Center. Male Wistar rats weighing 170-190 g (Harlan) were housed individually $\left(250 \mathrm{~cm}^{2} /\right.$ animal) and received food and water ad libitum. Animals were randomly assigned to the treatment or control group. All animals received a single subcutaneous injection of either monocrotaline ( $80 \mathrm{mg} / \mathrm{kg}$ body $\mathrm{wt}$ ) or saline. At 4 weeks after treatment, all animals were euthanized with a halothane overdose, and heart and lungs were excised. Hearts were rinsed by perfusion, and the LV and $\mathrm{RV}$ and septum were separated. All tissues were weighed and snap frozen in liquid nitrogen and stored at $-80^{\circ} \mathrm{C}$.

In vivo determination of T3-dependent transcriptional activity. A T3-responsive reporter vector containing $5 \mathrm{~T} 3$ receptor-binding half sites inserted upstream of the 141-bp skeletal muscle-specific SERCA1 minimal promoter (46) subcloned into the pGL3-basic Firefly luciferase reporter vector (Promega) was created using standard procedures and designated pLucTRE. The expression of a control vector containing the SERCA1 promoter without the T3 response element subcloned into the phRL-null Renilla luciferase reporter vector (Promega), was designated pRen-C and used in all experiments to normalize for variation in transfection efficiency. Rats were anesthetized with a mixture of $\mathrm{N}_{2} \mathrm{O}(0.2 \mathrm{l} / \mathrm{min}), \mathrm{O}_{2}(0.2 \mathrm{l} / \mathrm{min})$, and sevoflurane $(2 \%-3 \%)$. Hearts were exposed through a right-lateral thoracotomy, and, the RV and LV free wall were each injected 3 to 4 times using a 29 -gauge needle with a total volume of $100 \mu \mathrm{l}$ saline containing $10 \mu \mathrm{g}$ each of pLuc-TRE and pRen-C. The thorax was then closed, and rats were given an intraperitoneal injection of buprenorphine prior to recovery. Animals were sacrificed 5 days after direct gene transfer. Hearts were excised and rinsed by perfusion. The RV, LV, and septum were separated, weighed, snap frozen in liquid nitrogen, and then stored at $-80^{\circ} \mathrm{C}$. Tissue samples were homogenized in 2 volumes of luciferase lysis buffer (Promega) and centrifuged at $4^{\circ} \mathrm{C}$ for 15 minutes at $12,000 \mathrm{~g}$. The supernatant was analyzed in the combined Firefly and Renilla luciferase assay system (Promega). Reporter (pLuc-TRE) activity was reported as Firefly luciferase activity normalized to Renilla luciferase activity.

This method for determining T3-dependent transcriptional activity in the heart was validated by comparing euthyroid (control), hypothyroid, and T3-treated hypothyroid rats (male Wistar rats, weighing 170-190 g; Harlan). Hypothyroidism was induced by adding $2 \% \mathrm{KClO}_{4}$ to the drinking water for a period of 6 weeks (47). Plasma T3 levels were $0.027 \pm 0.004 \mathrm{nM}$ $(n=6)$ at the time of sacrifice, and T4 levels were undetectable. T3 treatment of hypothyroid rats $(20 \mu \mathrm{g} / 100 \mathrm{~g}$ body weight, daily subcutaneous injection) to induce hyperthyroidism was started 1 day before plasmid injections. Plasma T3 levels were $118 \pm 13 \mathrm{nM}(n=6)$, and T4 levels were $11.8 \pm 1.4 \mathrm{nM}(n=6)$ at the time of sacrifice (see Table 1 for control values). Hearts were transfected as described above, and luciferase activities were determined 5 days later. The average ventricular luciferase activity of Luc normalized to that of Ren (mean \pm SEM) was $2.7 \pm 0.2(n=29)$ for control, $1.2 \pm 0.2(n=5)$ for hypothyroid ( $P<0.008$ vs. control), and $20.7 \pm 1.4(n=9)$ for T3-treated rats $(P<0.0001$ vs. control). The T3-dependent transcriptional activity in the RV and LV of control and failing hearts was determined by coinjection of the plasmids into the myocardium 19 days after saline or monocrotaline administration.

Western blotting. Ventricular tissue was homogenized in Histidine/ KCl buffer (20 and $100 \mathrm{mM}$, respectively), and equal amounts of protein $(10 \mu \mathrm{g})$ were separated by electrophoresis $(20 \%$ SDS-PAGE) and transferred to nitrocellulose membranes. For tissue samples, equal protein transfer was confirmed by reversible Ponceau staining, and membranes were blocked with $5 \%$ nonfat milk and immunostained with antisera against HIF- $1 \alpha$ (Santa Cruz Biotechnology Inc.) or HIF-2 $\alpha$ (Novus Biologicals). Blots were incubated overnight at $4{ }^{\circ} \mathrm{C}$ in TBS containing $1 \%$ nonfat milk, and bands were visualized by enhanced chemiluminescence and quantified using a Fuji Film LAS 3000 laser densitometer (Fuji Medical Systems). Sonicates of isolated cells were prepared in $0.1 \mathrm{M}$ phosphate and $1 \mathrm{mM}$ EDTA at $\mathrm{pH} 6.9$ with $10 \mathrm{mM}$ dithiothreitol and $0.25 \mathrm{M}$ sucrose supplemented with Complete Mini protease inhibitor cocktail tablets (Roche), and Western blotting was performed with the BM chemiluminescence Western blotting kit (Roche). For NCLP6E cells, the ECL Plus Western Blotting Detection System (GE Healthcare) was used.

Quantitative real-time PCR. PCR primers were designed using Primer Express version 2.0 (Invitrogen). Expression levels of HPRT were used for normalization. A total of $5 \mu \mathrm{g}$ total RNA was used to generate cDNA strands in a 20- $\mu 1$ reaction volume using the Cloned AMV First Strand Synthesis Kit (Invitrogen). An equivalent of $25 \mathrm{ng}$ total RNA was subsequently used in the amplification with $50 \mathrm{nM}$ gene-specific primers and $4 \mu \mathrm{l} \mathrm{SYBR}$ green mix (Applied Biosystems) in a total volume of $8 \mu \mathrm{l}$, using standard cycle parameters on an Applied Biosystems model 7700.

Plasma and tissue thyroid hormone levels. Plasma T4 and T3 were determined by RIA as previously described (21). Tissue thyroid hormone concentrations were determined using methanol-chloroform extraction, back extraction into an aqueous phase, and purification of heart extracts on Dowex $1 \mathrm{X} 2$ columns as previously described (48). T3 and T4 content of the purified extracts was determined using sensitive RIAs, and the results were calculated using individual recovery data obtained by the addition of tracer amounts of $\left[{ }^{131} \mathrm{I}\right] \mathrm{T} 4$ and $\left[{ }^{125} \mathrm{I}\right] \mathrm{T} 3$ to the initial homogenates.

Statistics. Comparisons were performed by Student $t$ test, and multiple comparisons were by ANOVA followed by the Student-Newman-Keuls test. A $P$ value less than 0.05 was considered significant.

\section{Acknowledgments}

This work was supported by grant 903-40-194 from the Netherlands Organization for Scientific Research (NWO); grants DK76099, DK44128, and DK65055 from the National Institutes of Health; the Lawson Wilkins Abbott Clinical Scholar Award; the Charles A. King Trust Postdoctoral Research Fellowship Award; the Charles A. Janeway Child Health Research Center Award; and the Clinical Scientist Development Award 
from the Doris Duke Charitable Foundation. We thank Yujiang Shi for helpful insights and comments on this manuscript and on ChIP methodologies.

Received for publication May 29, 2007, and accepted in revised form December 5, 2007.

Address correspondence to: Stephen A. Huang, Harvard Institutes of Medicine, 77 Avenue Louis Pasteur, Room 642, Boston, Massa- chusetts 02115, USA. Phone: (617) 355-2452; Fax: (617) 730-0793; E-mail: stephen.huang@childrens.harvard.edu.

Wagner S. da-Silva's present address is: Instituto de Bioquímica Médica, Universidade Federal do Rio de Janeiro, Rio de Janeiro, Brazil.

Felix B. Engel's present address is: Max-Planck-Institute for Heart and Lung Research, Department for Heart Development and Regeneration, Bad Nauheim, Germany.
1. Langton, J.E., and Brent, G.A. 2002. Nonthyroidal illness syndrome: evaluation of thyroid function in sick patients. Endocrinol. Metab. Clin. North Am. 31:159-172.

2. Kaptein, E.M., Weiner,J.M., Robinson, W.J., Wheeler, W.S., and Nicoloff, J.T. 1982. Relationship of altered thyroid hormone indices to survival in nonthyroidal illnesses. Clin. Endocrinol. (Oxf.). 16:565-574.

3. Brent, G.A., and Hershman, J.M. 1986. Thyroxine therapy in patients with severe nonthyroidal illnesses and low serum thyroxine concentration. J. Clin. Endocrinol. Metab. 63:1-8.

4. Acker, C.G., Singh, A.R., Flick, R.P., Bernardini, J., Greenberg, A., and Johnson, J.P. 2000. A trial of thyroxine in acute renal failure. Kidney Int. 57:293-298.

5. De Groot, L.J. 1999. Dangerous dogmas in medicine: the nonthyroidal illness syndrome. J. Clin. Endocrinol. Metab. 84:151-164.

6. Utiger, R.D. 1980. Decreased extrathyroidal triiodothyronine production in nonthyroidal illness: benefit or harm? Am. J. Med. 69:807-810.

7. Bianco, A.C., and Kim, B.W. 2006. Deiodinases: implications of the local control of thyroid hormone action. J. Clin. Invest. 116:2571-2579.

8. Huang, S.A. 2005. Physiology and pathophysiology of type 3 deiodinase in humans. Thyroid. 15:875-881.

9. Richard, K., et al. 1998. Ontogeny of iodothyronine deiodinases in human liver. J. Clin. Endocrinol. Metab. 83:2868-2874.

10. Kester, M.H., et al. 2004. Iodothyronine levels in the human developing brain: major regulatory roles of iodothyronine deiodinases in different areas. J. Clin. Endocrinol. Metab. 89:3117-3128.

11. Peeters, R.P., et al. 2003. Reduced activation and increased inactivation of thyroid hormone in tissues of critically ill patients. J. Clin. Endocrinol. Metab. 88:3202-3211.

12. Marsh-Armstrong, N., Huang, H., Remo, B.F., Liu, T.T., and Brown, D.D. 1999. Asymmetric growth and development of the Xenopus laevis retina during metamorphosis is controlled by type III deiodinase. Neuron. 24:871-878.

13. Huang, S.A., et al. 2005. TGF- $\{$ beta\} promotes inactivation of extracellular thyroid hormones via transcriptional stimulation of type 3 iodothyronine deiodinase. Mol. Endocrinol. 19:3126-3136.

14. Huang, S.A., Dorfman, D.M., Genest, D.R., Salvatore, D., and Larsen, P.R. 2003. Type 3 iodothyronine deiodinase is highly expressed in the human uteroplacental unit and in fetal epithelium. J. Clin. Endocrinol. Metab. 88:1384-1388.

15. Lamirand, A., Mercier, G., Ramauge, M., Pierre, M., and Courtin, F. 2007. Hypoxia stabilizes type 2 deiodinase activity in rat astrocytes. Endocrinology. 148:4745-4753.

16. Hernandez, A., and Obregon, M.J. 1995. Presence of growth factors-induced type III iodothyronine 5 -deiodinase in cultured rat brown adipocytes. Endocrinology. 136:4543-4550.

17. Huang, L.E., and Bunn, H.F. 2003. Hypoxia-inducible factor and its biomedical relevance. J. Biol.
Chem. 278:19575-19578.

18. Curcio, C., et al. 2001. The human type 2 iodothyronine deiodinase is a selenoprotein highly expressed in a mesothelioma cell line. J. Biol. Chem. 276:30183-30187.

19. Shi, Y., et al. 2004. Histone demethylation mediated by the nuclear amine oxidase homolog LSD1. Cell. 119:941-953.

20. da-Silva, W.S., et al. 2007. The small polyphenolic molecule kaempferol increases cellular energy expenditure and thyroid hormone activation. Diabetes. 56:767-776.

21. Wassen, F.W., et al. 2002. Induction of thyroid hormone-degrading deiodinase in cardiac hypertrophy and failure. Endocrinology. 143:2812-2815.

22. Des Tombe, A.L., Van Beek-Harmsen, B.J., Lee-De Groot, M.B., and Van Der Laarse, W.J. 2002. Calibrated histochemistry applied to oxygen supply and demand in hypertrophied rat myocardium. Microsc. Res. Tech. 58:412-420.

23. Buermans, H.P., et al. 2005. Microarray analysis reveals pivotal divergent $\mathrm{mRNA}$ expression profiles early in the development of either compensated ventricular hypertrophy or heart failure. Physiol. Genomics. 21:314-323.

24. Acsadi, G., et al. 1991. Direct gene transfer and expression into rat heart in vivo. New Biol. 3:71-81.

25. Dean, D.A. 2005. Nonviral gene transfer to skeletal, smooth, and cardiac muscle in living animals. Am. J. Physiol. Cell Physiol 289:C233-C245.

26. Roti, E., Fang, S.L., Green, K., Emerson, C.H., and Braverman, L.E. 1981. Human placenta is an active site of thyroxine and 3,3'5-triiodothyronine tyrosyl ring deiodination. J. Clin. Endocrinol. Metab. 53:498-501.

27. Salvatore, D., et al. 1995. Type 3 lodothyronine deiodinase: cloning, in vitro expression, and functional analysis of the placental selenoenzyme. J. Clin. Invest. 96:2421-2430.

28. Becker, K.B., Schneider, M.J., Davey, J.C., and Galton, V.A. 1995. The type III 5-deiodinase in Rana catesbeiana tadpoles is encoded by a thyroid hormoneresponsive gene. Endocrinology. 136:4424-4431.

29. Huang, S.A., et al. 2000. Severe hypothyroidism caused by type 3 iodothyronine deiodinase in infantile hemangiomas. N. Engl. J. Med. 343:185-189.

30. Rankin, E.B., et al. 2007. Hypoxia-inducible factor2 (HIF-2) regulates hepatic erythropoietin in vivo. J. Clin. Invest. 117:1068-1077.

31. Stolze, I., et al. 2002. Hypoxia-inducible erythropoietin gene expression in human neuroblastoma cells. Blood. 100:2623-2628.

32. Calvani, M., Rapisarda, A., Uranchimeg, B., Shoemaker, R.H., and Melillo, G. 2006. Hypoxic induction of an HIF-1alpha-dependent bFGF autocrine loop drives angiogenesis in human endothelial cells. Blood. 107:2705-2712.

33. Baqui, M., et al. 2003. Human type 3 iodothyronine selenodeiodinase is located in the plasma membrane and undergoes rapid internalization to endosomes. J. Biol. Chem. 278:1206-1211.
34. Baqui, M.M., et al. 2000. Distinct subcellular localization of transiently expressed types 1 and 2 iodothyronine deiodinases as determined by immunofluorescence confocal microscopy. Endocrinology. 141:4309-4312.

35. Bianco, A.C., and Silva, J.E. 1988. Cold exposure rapidly induces virtual saturation of brown adipose tissue nuclear T3 receptors. Am. J. Physiol. 255:E496-E503.

36. Park, M.K. 2002. Fetal and perinatal circulation. In Pediatric cardiology for practitioners. M.K. Park, editor. Mosby. St. Louis, Missouri, USA. 93-97.

37. Koch, G., and Wendel, H. 1968. Adjustment of arterial blood gases and acid base balance in the normal newborn infant during the first week of life. Biol. Neonat. 12:136-161.

38. Moshang, T., Jr., Chance, K.H., Kaplan, M.M., Utiger, R.D., and Takahashi, O. 1980. Effects of hypoxia on thyroid function tests. J. Pediatr. 97:602-604.

39. Silva, J.E., and Larsen, P.R. 1977. Pituitary nuclear 3,5,3'-triiodothyronine and thyrotropin secretion: an explanation for the effect of thyroxine. Science. 198:617-620.

40. Silva, J.E., Leonard, J.L., Crantz, F.R., and Larsen, P.R. 1982. Evidence for two tissue specific pathways for in vivo thyroxine 5 'deiodination in the rat. J. Clin. Invest. 69:1176-1184.

41. de Jesus, L.A., et al. 2001. The type 2 iodothyronine deiodinase is essential for adaptive thermogenesis in brown adipose tissue. J. Clin. Invest. 108:1379-1385.

42. Tu, H.M., et al. 1999. Regional expression of the type 3 iodothyronine deiodinase messenger ribonucleic acid in the rat central nervous system and its regulation by thyroid hormone. Endocrinology. 140:784-790.

43. Hernandez, A., Martinez, M.E., Fiering, S., Galton, V.A., and St Germain, D. 2006. Type 3 deiodinase is critical for the maturation and function of the thyroid axis. J. Clin. Invest. 116:476-484.

44. Engel, F.B., et al. 2005. p38 MAP kinase inhibition enables proliferation of adult mammalian cardiomyocytes. Genes Dev. 19:1175-1187.

45. Callebaut, I., et al. 2003. The iodothyronine selenodeiodinases are thioredoxin-fold family proteins containing a glycoside hydrolase clan GH-A-like structure. J. Biol. Chem. 278:36887-36896.

46. Simonides, W.S., et al. 1996. Characterization of the promoter of the rat sarcoplasmic endoplasmic reticulum Ca2+-ATPase 1 gene and analysis of thyroid hormone responsiveness. J. Biol. Chem. 271:32048-32056.

47. Simonides, W.S., and van Hardeveld, C. 1986. Effects of the thyroid status on the sarcoplasmic reticulum in slow skeletal muscle of the rat. Cell Calcium. 7:147-160.

48. Calvo, R., Obregon, M.J., Escobar del Rey, F., and Morreale de Escobar, G. 1992. The rat placenta and the transfer of thyroid hormones from the mother to the fetus. Effects of maternal thyroid status. Endocrinology. 131:357-365. 